\title{
SEGMENTING A BEATING HEART USING POLYSEGMENT AND SPATIAL GPCA
}

\author{
Avinash Ravichandran ${ }^{1}$, René Vidal ${ }^{1}$ and Henry Halperin ${ }^{2}$ \\ ${ }^{1}$ Center for Imaging Science, Johns Hopkins University, USA \\ ${ }^{2}$ Cardiovascular Medicine, Johns Hopkins School of Medicine, USA
}

\begin{abstract}
Given a volume of cardiac MR images, we consider the problem of segmenting the heart based on intensity and dynamics. We first segment the heart and the chest from the background using an algebraic technique for intensity based segmentation called Polysegment. As the heart and the chest exhibit different dynamics, we model the image temporal evolution as the output of two different linear dynamical systems. Under this model, the trajectories of the heart and chest intensities lie in different subspaces. We thus propose a method called Spatial GPCA for clustering data points lying in multiple subspaces, while maintaining the spatial coherence of the data points. We compare the segmentation results of Spatial GPCA to those of K-Subspaces, another popular subspace clustering algorithm.
\end{abstract}

\section{INTRODUCTION}

Building a 3-D model of the heart is potentially very useful for many clinical procedures, e.g., radio-frequency ablation. Currently most of the visualization for such procedures is done by either 2-D fluoroscopy or manual segmentation from 3-D CT models. Since fluoroscopy gives a 2-D projection of the 3-D object, it is difficult to accurately determine a 3-D location. On the other hand, manual segmentation is time consuming and suffers from reliability and consistency issues.

In order to build a 3-D model for clinicians to use, the first step is to segment the heart from other regions of the MR volume. Segmentation based on intensity only is bound to fail, as the intensities of the heart and chest are not very different. Likewise, traditional rigid body motion segmentation methods would fail, because the heart behaves as a non-rigid body deforming in a periodic manner according to the cardiac cycle. A simple heart segmentation method using both intensity and dynamics is to threshold the temporal standard deviation of the intensity at each voxel [1]. However, the method fails with noisy sequences, as we will show in the experiments.

In this paper, we propose to model the temporal evolution of a beating heart as a dynamic texture [2], i.e. as the output of a time invariant linear dynamical system, and to segment such a dynamic texture using subspace clustering techniques. Previous work on dynamic texture segmentation [3] is based on minimizing a Mumford-Shah like cost functional within a level-set framework. However, level-set techniques are usually very computationally intensive. Since our long term goal is to design a real-time system, in this paper we propose an algebraic technique for dynamic texture segmentation and subspace clustering called Spatial GPCA. The advantage over iterative techniques being that algebraic techniques are less computationally intensive and do not need initialization.

\section{INTENSITY BASED SEGMENTATION}

MR images of the heart consist of three main regions: heart, chest and background (which is predominantly noise). Since the dynamics caused by variation of noise across images will influence the segmentation, we first remove the background noise using an intensity based segmentation method.

Currently, there is a huge body of literature on intensity based image segmentation, including algorithms such as KMeans and Expectation Maximization (EM) [4]. Since our long term objective is to develop a real-time system, we use an algebraic approach to image segmentation, called Polysegment. As the method is algebraic and non-iterative, it is faster than EM or K-Means and does not require any initialization.

Polysegment performs image segmentation by fitting the image intensities with a polynomial whose roots give the mean intensity of each region. The algorithm proceeds as follows. Let $\boldsymbol{x} \in \mathbb{R}^{P}$ be a given vector containing the image intensities associated with $P$ pixels or voxels. We would like to segment $\boldsymbol{x}$ into $n$ groups, each one having an unknown intensity value $V_{i}, i=1, \ldots, n$, where $V_{1} \neq V_{2} \neq \cdots \neq V_{n}$. To this end, let $x$ be an arbitrary entry of $x$. Then, there exists a constant $V_{i}$ such that $x=V_{i}$. This means that

$$
\left(x=V_{1}\right) \vee\left(x=V_{2}\right) \vee\left(x=V_{n}\right),
$$

which can be compactly written as the following polynomial of degree $n$ in $x$

$$
p_{n}(x)=\prod_{i=1}^{n}\left(x-V_{i}\right)=\sum_{k=0}^{n} c_{k} x^{k}=0 .
$$

Since equation (2) is valid for every entry of $\boldsymbol{x}$, we can solve for the vector of coefficients $c \in \mathbb{R}^{n+1}$ from

$$
L_{n} \boldsymbol{c} \doteq\left[\begin{array}{ccccc}
1 & x_{1} & x_{1}^{2} & \cdots & x_{1}^{n} \\
1 & x_{2} & x_{2}^{2} & \cdots & x_{2}^{n} \\
\vdots & & & & \vdots \\
1 & x_{P} & x_{P}^{2} & \cdots & x_{P}^{n}
\end{array}\right]\left[\begin{array}{c}
c_{0} \\
\vdots \\
c_{n-1} \\
1
\end{array}\right]=0
$$

where $L_{n} \in \mathbb{R}^{P \times(n+1)}$ is the data matrix. 
Given $\boldsymbol{c}$, we can obtain $\left\{V_{i}\right\}_{i=1}^{n}$ as the $n$ roots of the polynomial $p_{n}(x)$. Given $\left\{V_{i}\right\}_{i=1}^{n}$, the segmentation is obtained by assigning point $j$ to group $i$ whenever

$$
i=\arg \min _{k=1, \cdots, n}\left(x_{j}-V_{k}\right)^{2} .
$$

\section{DYNAMICS BASED SEGMENTATION}

\subsection{Modeling the Heart Motion as a Dynamic Texture}

On examining the temporal evolution of cardiac MR images from a particular spatial location, we observe that the chest does not exhibit any dynamics while the heart deforms according to the cardiac cycle. Thus, we propose to model a video sequence of a beating heart, $I(t) \in \mathbb{R}^{P}, t \in\{1 \cdots F\}$, as a dynamic texture, i.e. as the output of a linear dynamical system (LDS), which models both the appearance at every frame and the dynamics of all the frames. As proposed in [5], we model a dynamic texture with an ARMA model of order $d$

$$
\begin{aligned}
z(t+1) & =A z(t)+B \eta(t) \\
I(t) & =C z(t)+w(t)
\end{aligned}
$$

where $A \in \mathbb{R}^{d \times d}, B \in \mathbb{R}^{d \times d_{\eta}}, C \in \mathbb{R}^{P \times d}, \eta(t) \in \mathbb{R}^{d_{\eta}}$ is the process noise and $w(t) \in \mathbb{R}^{P}$ is the measurement noise.

If the scene has only one dynamic texture, one can learn the parameters $(A, B, C)$ optimally and in closed form using subspace identification techniques such as N4SID [6]. However, such techniques are computationally intensive when applied to video sequences, because the number of pixels is typically very large. A sub-optimal solution can be obtained by noticing that when $\eta=w=0$ the video sequence can be stacked into a matrix $W \in \mathbb{R}^{P \times F}$ which can be written as

$$
W=[I(1) \cdots I(F)]=C[z(1) \cdots z(F)]=C Z .
$$

Therefore, $\operatorname{rank}(W) \leq d \leq F, P$, and one can learn $C$ and $Z$ from the singular value decomposition of $W$. Given $Z$, solving for $A$ is a linear problem. Given $A$ and $Z$, solving for $B$ (assuming now that $\eta \neq 0$ ) is also a linear problem $[2,5]$.

In the case of an MR volume of the heart, the scene is composed of two dynamic textures: one for the chest and one for the heart. Therefore, each row of $W$ lives approximately in one out of two possible subspaces of $\mathbb{R}^{F}$ of dimension at most $d$. The problem of segmenting the heart from the chest is then equivalent to one of clustering the rows of $W$ into two subspaces. This leads us to the idea of using subspace clustering algorithms such as K-Subspaces [7] or Generalized Principal Component Analysis (GPCA) [8] to segment the two regions of the MR images.

However, neither GPCA nor K-Subspaces incorporate the fact that nearby voxels should belong to the same group. Thus, due to the presence of noise in the image, the segmentation can have misclassification of isolated points. To overcome this issue, we propose a modification to the GPCA algorithm, which we dub Spatial GPCA, that incorporates spatial regularization into the segmentation process.

\subsection{Spatial GPCA}

Spatial GPCA is an algebraic subspace clustering algorithm that computes a normal to the subspace at a data point from the derivatives of a polynomial that is fitted to all data points. Once the normals are known, the data points are clustered based on their distance to each subspace. More specifically, let $\boldsymbol{w}$ be any of the rows of $W$. Let $S_{c}=\left\{\boldsymbol{w}: \boldsymbol{b}_{c}^{T} \boldsymbol{w}=0\right\}$ and $S_{h}=\left\{\boldsymbol{w}: \boldsymbol{b}_{h}^{T} \boldsymbol{w}=0\right\}$ be the subspaces associated with the chest and heart with normals $\boldsymbol{b}_{c}$ and $\boldsymbol{b}_{h}$, respectively. The Spatial GPCA algorithm operates as follows:

1. Project the rows of $W \in \mathbb{R}^{P \times F}$ onto a low-dimensional subspace of dimension $d+1$. This can be done by first computing the SVD of $W=U S V^{T}$, and then letting $W_{p} \in \mathbb{R}^{P \times(d+1)}$ be the first $d+1$ columns of $U$.

2. Fit a polynomial to the rows of $W_{p}$. The rationale behind this step is as follows. Let $\boldsymbol{w} \in \mathbb{R}^{d+1}$ be any of the rows of $W_{p}$. Since $\boldsymbol{w}$ must belong to one of the two projected subspaces, say $S_{k}$, then there exists a vector $\boldsymbol{b}_{k} \in \mathbb{R}^{d+1}$ normal to $S_{k}$ such that $\boldsymbol{b}_{k}^{T} \boldsymbol{w}=0$. Then any row $\boldsymbol{w}$ of $W_{p}$ must satisfy the following homogeneous polynomial of degree two in $d+1$ variables

$$
p(\boldsymbol{w})=\left(\boldsymbol{b}_{h}^{T} \boldsymbol{w}\right)\left(\boldsymbol{b}_{c}^{T} \boldsymbol{w}\right)=0 .
$$

This polynomial can be expressed linearly in terms of its coefficients. For instance, if $d=1$ we have $p(\boldsymbol{w})=$ $c_{1} w_{1}^{2}+c_{2} w_{1} w_{2}+c_{3} w_{2}^{2}$, hence we can solve for the coefficients of $p$ from the linear system

$$
\left[c_{1}, c_{2}, c_{3}\right]\left[\begin{array}{ccc}
w_{11}^{2} & \cdots & w_{1 P}^{2} \\
w_{11} w_{21} & \cdots & w_{1 P} w_{2 P} \\
w_{21}^{2} & \cdots & w_{2 P}^{2}
\end{array}\right]=0 .
$$

3. Solve for the normal vectors from the derivatives of $p$. This is because the polynomial $p=0$ describes a surface in $\mathbb{R}^{d+1}$, i.e. the union of the 2 projected subspaces. Therefore, the derivative of $p$ at a point gives a vector normal to the subspace passing through that point, i.e.

$$
\boldsymbol{b}_{k}=\frac{D p(\boldsymbol{w})}{\|D p(\boldsymbol{w})\|} .
$$

4. Compute $D p(\boldsymbol{w})$ in a neighborhood $\Omega(\boldsymbol{x})$ of size $\tau$ around each voxel $\boldsymbol{x}$. Since all voxels in $\Omega(\boldsymbol{x})$ are likely to belong to the same group, we "smooth" the derivatives by replacing the derivative of $p$ at $\boldsymbol{x}$ by the principal component of all the derivatives of $p$ at voxels in $\Omega(\boldsymbol{x})$. Before computing the principal component, one can also scale the derivatives in $\Omega(\boldsymbol{x})$ by a Gaussian function, so as to give more weight to the central voxel $\boldsymbol{x}$.

5. Once the derivatives are "smoothed", the point $\boldsymbol{w}$ is assigned to group $i$ where

$$
i=\arg \min _{k=h, c}\left\{\left(\boldsymbol{b}_{k}^{T} D p(\boldsymbol{w})\right)^{2}\right\} .
$$




\subsection{K-Subspaces}

K-Subspaces is an iterative method for segmenting data lying in multiple subspaces. Given an initial estimate for the subspace bases, this algorithm alternates between clustering the data points using the distance residual to the different subspaces, and computing a basis for each subspace using standard PCA. The K-Subspaces algorithm is outlined below.

1. Set $m=0$ and randomly initialize the bases for the subspaces $S_{h}$ and $S_{c}$ with orthonormal matrices $U_{h}^{(0)} \in$ $\mathbb{R}^{(d+1) \times d}$ and $U_{c}^{(0)} \in \mathbb{R}^{(d+1) \times d}$.

2. Assign each point $\boldsymbol{w}_{j}$ to group $i$ (heart or chest) if

$$
i=\arg \min _{k=h, c}\left\|\boldsymbol{w}_{j}-U_{k}^{(m)}\left(U_{k}^{(m)}\right)^{T} \boldsymbol{w}_{j}\right\|^{2} .
$$

Let $\boldsymbol{w}_{h}^{(m)}$ be the set of points associated with the heart, and $\boldsymbol{w}_{c}^{(m)}$ the set of points associated with the chest.

3. Calculate a basis for each subspace $U_{h}^{(m+1)}$ and $U_{c}^{(m+1)}$

$$
U_{h}^{(m+1)}=P C A\left(\boldsymbol{w}_{h}^{(m)}\right), U_{c}^{(m+1)}=P C A\left(\boldsymbol{w}_{c}^{(m)}\right) .
$$

4. If $\boldsymbol{w}_{h}^{(m+1)}=\boldsymbol{w}_{h}^{(m)}$ and $\boldsymbol{w}_{c}^{(m+1)}=\boldsymbol{w}_{c}^{(m)}$ or $m>m_{\max }$ stop. Else, set $m=m+1$ and goto step 2 .

As the convergence of K-Subspaces to the global optimum depends strongly on correct initialization, one usually starts the algorithm from multiple initializations. One can avoid this issue by using the result of Spatial GPCA to initialize K-Subspaces.

\section{EXPERIMENTAL RESULTS}

We applied the proposed segmentation technique to a data set consisting of 20 temporal volumes from 9 spatial locations. For computational efficiency, we applied the algorithm to each spatial slice and downsampled the images from $256 \times$ 256 to $128 \times 128$.

Figure 1 shows intensity based segmentation results using Polysegment and K-Means [4]. The results show that both algorithms perform equally well, though Polysegment is fractionally faster and does not require any initialization.

After removing the background using intensity based segmentation, we applied Spatial GPCA and GPCA (same as Spatial GPCA, but with step 4 omitted) to one spatial slice. Figure 2 shows the segmentation results from both algorithms. Note that the segmentation from the GPCA algorithm is not spatially coherent with a lot of isolated points getting misclassified. The segmentation from the Spatial GPCA algorithm gives a spatially more coherent segmentation, particularly for the chest, thanks to the spatial regularization step. Figure 3 shows more heart segmentation results for a few other slices.

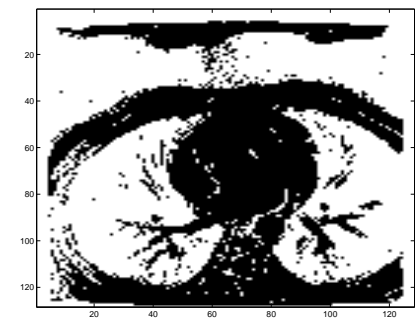

(a) Polysegment

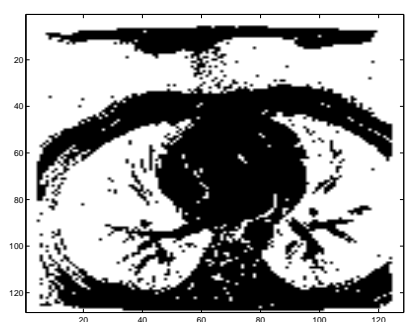

(b) K-Means
Fig. 1. Intensity based segmentation with $n=15$ groups: white corresponds to the group of lowest intensity (background) while white corresponds to all remaining groups.

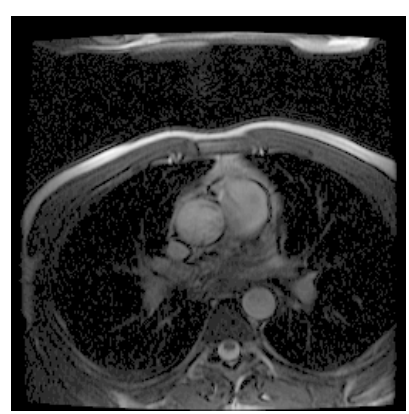

(a) Original image
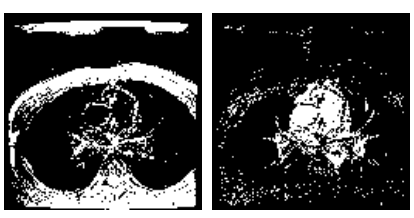

(b) GPCA segmentation
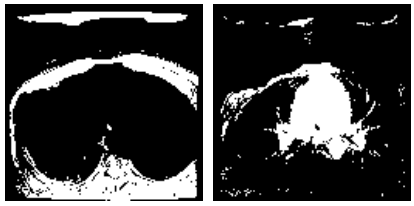

(c) Spatial GPCA segmentation
Fig. 2. Dynamics based segmentation using GPCA. (a) shows one frame of the sequence, (b) shows the points segmented as chest and heart using GPCA and (c) shows the segmentation using Spatial GPCA with a window $\Omega$ of $\operatorname{size} \tau=3$.
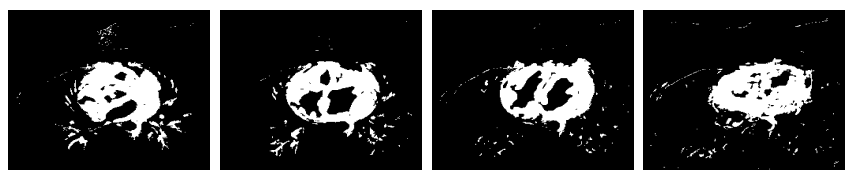

Fig. 3. Spatial GPCA results showing the heart segmentation for four other slices of the heart.

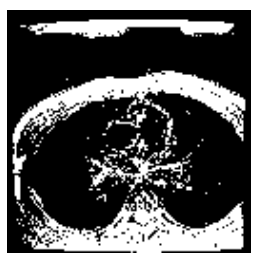

GPCA

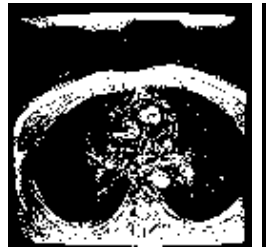

K-Subspaces

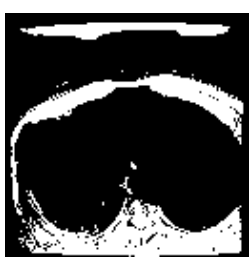

Spatial GPCA
Fig. 4. Comparison of chest segmentation for a particular spatial location.

Figure 4 shows the performance of all three algorithms on one spatial location. Notice that spatial GPCA gives a spatially more coherent segmentation of the heart than that of 
GPCA and K-Subspaces. However, the spatial regularization step in Spatial GPCA causes some regions of the chest near the heart to be classified as heart.

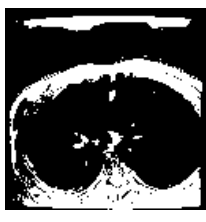

$\tau=3$

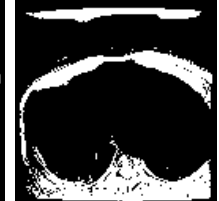

$\tau=9$

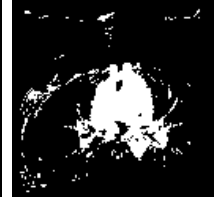

$\tau=3$

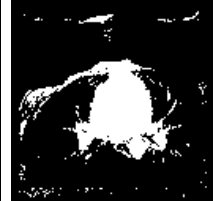

$\tau=9$
Fig. 5. Effect of the window size $\tau$ on dynamics based segmentation using Spatial GPCA.

We then studied the effect of varying the parameters of Spatial GPCA (window size and normal scaling function) on the quality of segmentation. Figure 5 shows the segmentation for a window size of $\tau=3$ and $\tau=9$. A larger window $\Omega$ gives a spatially more coherent segmentation at the cost of classifying parts of the chest as heart. We observed that scaling the normals in $\Omega$ with a Gaussian window did not have a noticeable effect on the segmentation.

A similar analysis was performed with the parameters of K-Subspaces (number of iteration and initialization). The number of iteration for K-subspaces was varied as $m_{\max }=$ $100,400,600$. Varying $m_{\max }$ did not have a noticeable effect on the segmentation. However, initializing K-subspaces with Spatial GPCA produced better results, than K-subspaces randomly initialized.

We also compared our results to a simple method based on thresholding the temporal standard deviation of the intensities at each pixel [1]. Figure 6(a) shows the results of the method for a spatial slice. Notice that the segmentation is fractionally better than that of Spatial GPCA shown in Figure 2(b). Figures 6(b)-(d) show segmentation results for another dataset in which the Spatial GPCA method outperforms the standard deviation method. One main difference between the two datasets is the amount of noise in each image. At this point we are tempted to make the claim that the Spatial GPCA based algorithm is more robust to noise. Nevertheless, we feel that more analysis is needed before we make the claim. These results, however, show a promising direction.

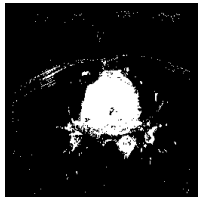

(a)

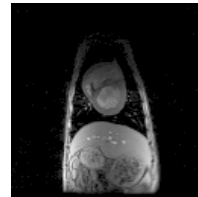

(b)

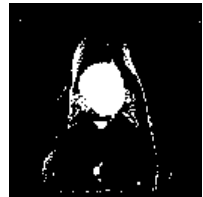

(c)

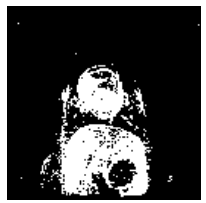

(d)
Fig. 6. Comparison of Spatial GPCA versus Standard Deviation Segmentation for the 2 datasets. (a) Standard deviation segmentation of the first dataset. (b) Sample image from the second dataset. (c) Dynamics based segmentation and the (d) Standard deviation-based segmentation.

\section{SUMMARY AND CONCLUSIONS}

We have proposed an algorithm for segmenting the heart from a 3-D cardiac MR volume. By modeling the temporal evolution of the intensities of each region with a linear system, we solve the segmentation problem using subspace clustering. Our approach, called Spatial GPCA, is algebraic and does not require initialization, making is suitable for real-time applications. Spatial GPCA also incorporates spatial regularization, which results in a spatially more coherent segmentation. The drawback of the algorithm, however, is that since it relies on the dynamics for the segmentation, if a region of the heart is static in an image sequence, it will be classified as the chest. In such cases, we propose that the dynamics based algorithm be used as an initialization for other methods.

\section{ACKNOWLEDGEMENTS}

Work supported by grant NIH-NHLBI 5-R01-HL-64795-01. We would like to thank Ahmet Genc and Muz Zviman for their help in data collection and for their feedback and insight.

\section{REFERENCES}

[1] U. Aladl, G. Hurwitz, D. Dey, D. Levin, M. Drangova, and P. Slomka, "Automated image registration of gated cardiac single-photon emission computed tomography and magnetic resonance imaging.," Journal of Magnetic Resonance Imaging, vol. 19, no. 3, pp. 283-290, 2004.

[2] G. Doretto, A. Chiuso, Y. Wu, and S. Soatto, "Dynamic textures," International Journal of Computer Vision, vol. 51, no. 2, pp. 91-109, 2003.

[3] G. Doretto, D. Cremers, P. Favaro, and S. Soatto, "Dynamic texture segmentation," in IEEE Conference on Computer Vision, 2003, pp. 44-49.

[4] R. Duda, P. Hart, and D. Stork, Pattern Classification, Wiley, New York, 2nd edition, 2000.

[5] S. Soatto, G. Doretto, and Y. Wu, "Dynamic textures," in IEEE International Conference on Computer Vision, 2001, pp. 439-446.

[6] B. De Moor, P. Van Overschee, and J. Suykens, "Subspace algorithms for system identification and stochastic realization," Tech. Rep. ESAT-SISTA Report 1990-28, Katholieke Universiteit Leuven, 1990.

[7] J. Ho, M.-H. Yang, J. Lim, K.-C. Lee, and D. Kriegman, "Clustering apperances of objects under varying illumination conditions," in IEEE Conference on Computer Vision and Pattern Recognition, 2003, vol. 1, pp. 11-18.

[8] R. Vidal, Y. Ma, and S. Sastry, "Generalized Principal Component Analysis (GPCA)," IEEE Trans. on Pattern Analysis and Machine Intelligence, vol. 27, no. 12, pp. 1945-1959, 2005. 